\title{
CONF-8708117--4
}

CONF-8708117--4

DE87 013539

\section{MAGNETIC DIFFUSE SCATTERING}

\author{
J. W. Cable \\ Oak Ridge National Laboratory \\ Solid State Division \\ P.O. Box X \\ Oak Ridge, Tennessee 37831 \\ U.S.A.
}

\section{DISCLAIMER}

This report was prepared as an account of work sponsored by an agency of the United States Government. Neither the United States Government nor any agency thereof, nor any of their employees, makes any warranty, express or implied, or assumes any legal liability or responsibility for the accuracy, completeness, or usefulness of any information, apparatus, product, or process disclosed, or represents that its use would not infringe privately owned rights. Reference herein to any specific commercial product, process, or service by trade name, trademark, manufacturer, or otherwise does not necessarily constitute or imply its endorsement, recommendation, or favoring by the United States Government or any agency thereof. The views and opinions of authors expressed herein do not necessarily state or reflect those of the United States Government or any agency thereof.
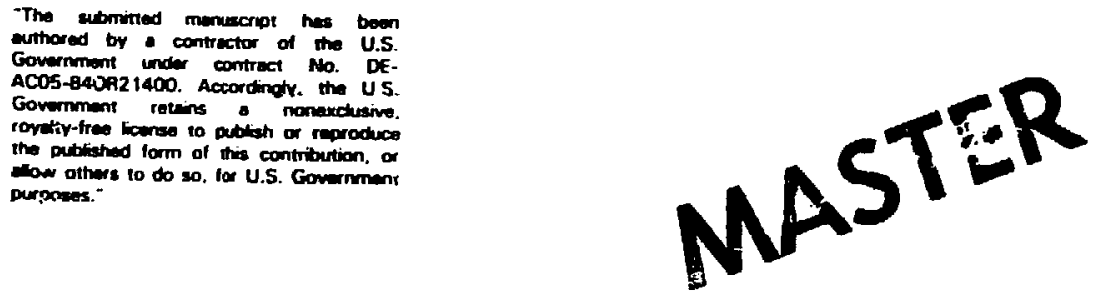


\author{
J. W. Cable \\ Oak Ridge National Laboratory \\ Solid State Division \\ P.O. Box X \\ Oak Ridge, Tennessee 37831 \\ U.S.A.
}

\title{
Abstract
}

The diffuse scattering of neutrons from magnetic materials provides unique and important information regarding the spatial correlations of the atoms and the spins. Such measurements have been extensively applied to magnetically ordered systems, such as the ferromagnet1c binary alloys, for which the observed correlations describe the magnetic moment fluctuations associated with local environment effects. With the advent of polarization analysis, these techniques are increasingly being applied to study disordered paramagnetic systems such as the spin-glasses and the diluted magnetic semiconductors. The spin-pair correlations obtained are essential in understanding the exchange interactions of such systems. In th1s paper, we describe recent neutron diffuse scattering results on the atompair and spin-pair correlations in some of these disordered magnetic systems.

\section{Introduction}

While the Bragg scattering from a crystal is determined by the average scattering density and the average lattice, the diffuse scattering is related to the fluctuations of the scattering system from those averages. Both types of scattering are required for understanding the structurs of real crystals, for which the diffuse scattering provides information on lattice distortions and on the spatial correlations of the atoms and the spins. In the case of magnetic materials, the nuclear and magnetic scattering often occurs in the same region of momentum space so that some separation technique 18 necessary. This separation method depends on the type of magnetic system being investigated. For a ferromagnet, Halperin and Johnson [1] showed that the magnetic scattering depends on both the Initial spin state of the neutron and on the prientation of the magnetic moment relative to the momentum transfer yector, $Q$. In particular, only that component of the moment perpendicular to $\vec{Q} 18$ effective In magnetic scattering. Th1s early theoretical result led to the development of two separation techniques for measurement of the magnetic diffuse scattering from ferromagnets. The first of these uses unpolarized neutrons and an applied magnetic field to extinguish the magnetic scattering by aligning the magnetic moments parallel to $\hat{Q}$. The second technique involves the use of polarized neutrons for which the nuclear and magnetic scattering amplitudes superpose. The scattered intensity therefore contains an additional nuclearmagnetis cross term which 18 readily separated by taking the difference between cross sections for incident beam polarizations parallel and antiparallel to the sample magnetization. This cross term is directly related to the effects of local environment on the magnetic moment distribution of the ferromagnetic alloy. Of course, nelther of these techniques can be used for those 
magnetic materials, such as the antiferromagnets and paramagnets, for which the magnetization can not be controlled by an external magnetic field. Polarization analysis has become the standard separation technique for such systems. Excellent reviews [2-4] are avallable which cover the magnetic diffuse scattering from ferromagnets and antiferromagnets. In this paper, we concentrate on the diffuse scattering from paramagnetic systems. This is a wellestablished area of research with a theoretical basis extending back to Halperin and Johnson [1] In 1939 and with the first neutron experiments performed by Shull, Strauser, and Wollan [5] In 1951. No survey of the vast IIterature on this subject is attempted; Instead the techniques and type of results obtalned are 1llustrated by presentation of a few recent studies carried out at oak Ridge.

\section{Diffuse Scattering from Cu-Mn Alloys}

The complicated magretic behavior of Cu-Mn alloys has attracted considerable attention for a long time [6]. The Cu-rich alloys exhibit typical spin glass behavior in wich the spins apparently freeze in random orlentations below a concentration-dependent freezing temperature, $T_{f}$. The magnetic susceptibility Is strongly dependent on heat treatment which suggests the presence of competing, short-range interactions between the Mn spins. Many neutron diffuse scattering studies [7-21] have been directed toward the determination of the 8pla-pair correlations that result from those interactions. In this fcc alloy, some degree of atomic short range order (ASRO) is always present and, unfortunately, the diffuse scattering arising from this ASRO superposes on the magnetic diffuse scattering over a large concentration region. This circumstance led to conflicting conclusions from some of the early neutron studies, but this has now been sorted out by use of polarization analysis and single crystal samples. The general features of the diffuse scattering from Cu-Mn alloys are illustrated by fIgure 1 which shows isolntensity contours in a [001] reciprocal lattice plane for a Cu-35\% $\mathrm{Mn}$ alloy. These room temperature data were taken on the WAND spectrometer at 0ak Ridge which uses an incident neutron energy of 35 mev with no energy analysis of the scattered beam. W1thIn the region covered by this scan, the allowed fundamental Bragg reflections are (200), (400), and (420). These reflections appear in the figure with Intensities that are off-scale and with spurious, comet-11ke talls directed back toward the origin along the scattering arc. The diffuse siattering appears in the diffuse peaks centerel $-:(11 / 20)$ type positions. The symmetry of this diffuse scattering distribution repeats in each Brillouin zone, but there is more intensity in the Inner zones because of a superposition of the nuclear and magnet1c scattering and the form factor dependence of the latter.

In favorable cases, a separation of this nuclear and magnetic scattering can be accomplished by taking temperature differences, but the best method is by polarization analysis. This technique [22] employs an incident neutron beam polarized in the $(+)$ direction and an analyzer-detector system that accepts neutrons only in the $(t)$ spin state. It is then possible to measure cross sections for $(++),(--),(+-)$ and $(-+)$ scattering by various on-off combinations of $\pi$ spin flippers located before and after the sample. If the experiment is arranged wth a guide field at the sample which maintains the 


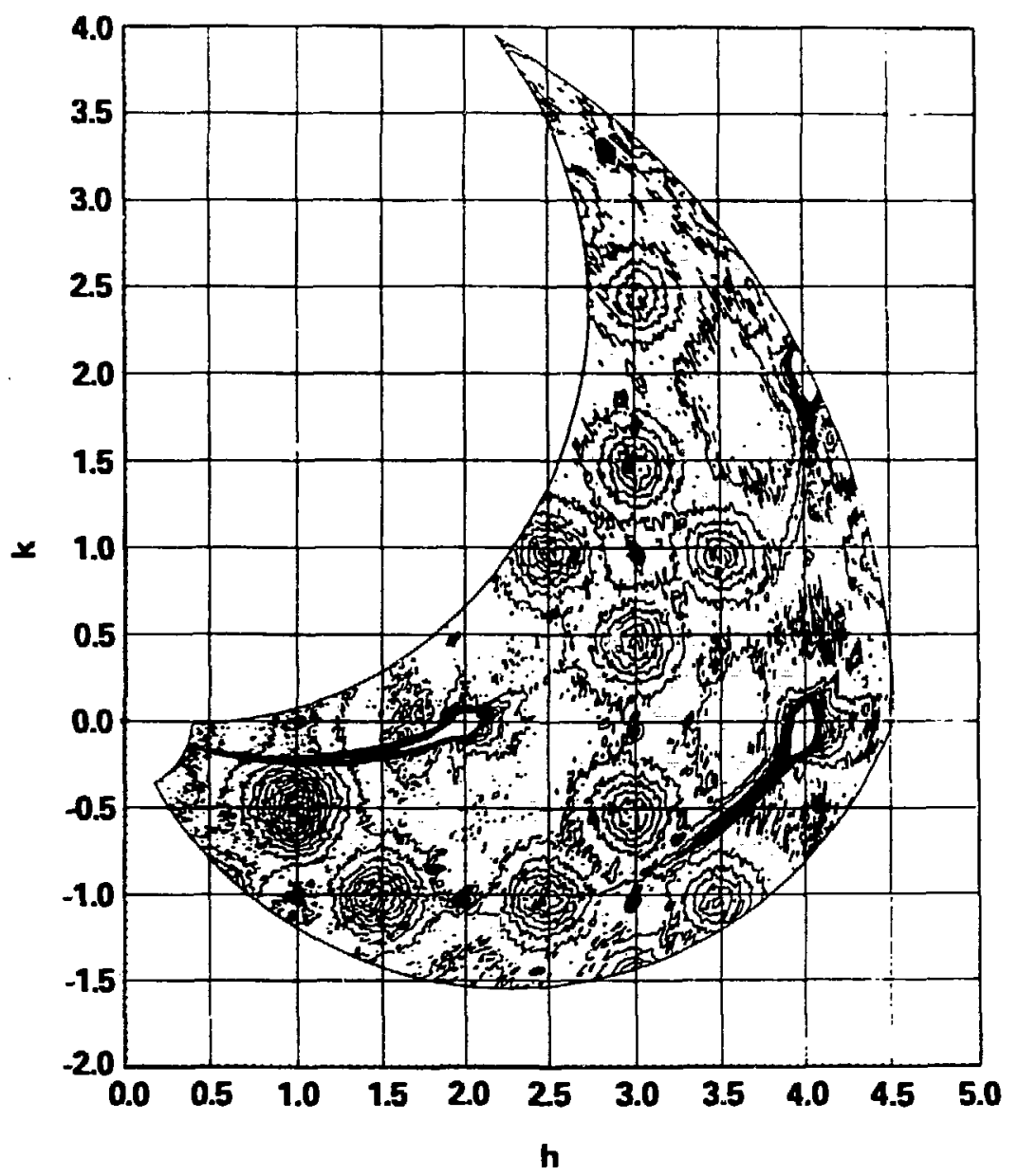

Figure 1. Isolntensity contours for an annealed Cu-35\% Mn alloy at $295 \mathrm{~K}$.

neutron polarization parallel to $\vec{Q}$, then the nuclear scattering is non-spinflip, $(t+)$ or $(--)$, while the magnetic scattering undergoes a spir-f11p, $(-+)$ or $(+-)$.

The non-spin-flip, or nuclear, cross section is given by

$$
\frac{d \sigma^{++}}{d \Omega}(\stackrel{Q}{Q})=c(1-c)\left(b_{1}-b_{h}\right)^{2} s(\mathbb{Q})
$$

where

$$
s(\mathbb{Q})=\sum_{\vec{R}} e^{1 \mathbb{Q} \cdot \vec{R}} \alpha(\mathbb{R})
$$

In which $b_{1}$ and $b_{h}$ are the Impur1ty and host nuclear scattering amplitudes, $c$ Is the Impurity concentration and the $\alpha(R)$ are the ASRo parameters. If $p_{n}^{+}$is 
defined as a site occupation operator that counts the number (e1ther 0 or 1) of impurity atoms at the lattice site $\vec{n}$, then

$$
c(1-c) \alpha(\vec{R})=\left\langle\left(p_{n}^{+}+\vec{R}-c\right)\left(p_{n}^{+}-c\right)\right\rangle
$$

where the angular brackets denote a configurational average. Thus, $\alpha\left({ }^{+}\right)$ describes the spatial correlation of site occupation fluctuations from the average.

The neutrov scattering cross section from an exchange coupled spin system is the Fourler transform of a time-dependent spin-pair correlation. Diffuse scattering experiments are ususally designed for quasi-elastic measurements which determine the static correlation for short times. Within the quasielastic approximation, the spin-flip, or magnetic, cross section for such a paramagnetic eystem can be written as

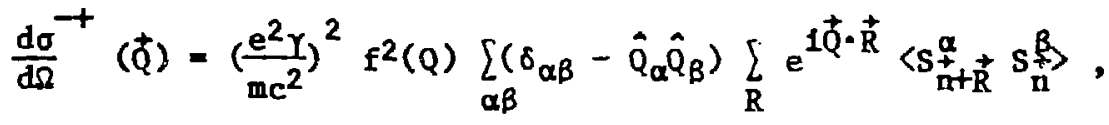

where $\alpha$ and $\beta$ are Cartesian coordinates, $\hat{Q}_{\alpha}$ and $\hat{Q}_{\beta}$ are direction cosines of $\vec{Q}^{\circ}$ and $\left\langle S_{n+R}^{\alpha+} S_{n}^{\beta}\right\rangle$ is the conflguratjonally averaged spin-pair correlation. Two conditions are required for this approximation to be valid: (1) the energy window of the analyzer-detector system must be wide enough to integrate over all energy transfers of the spln system, and (2) the incident energy must be sufficiently high that this integration 18 accomplished at nearly constant
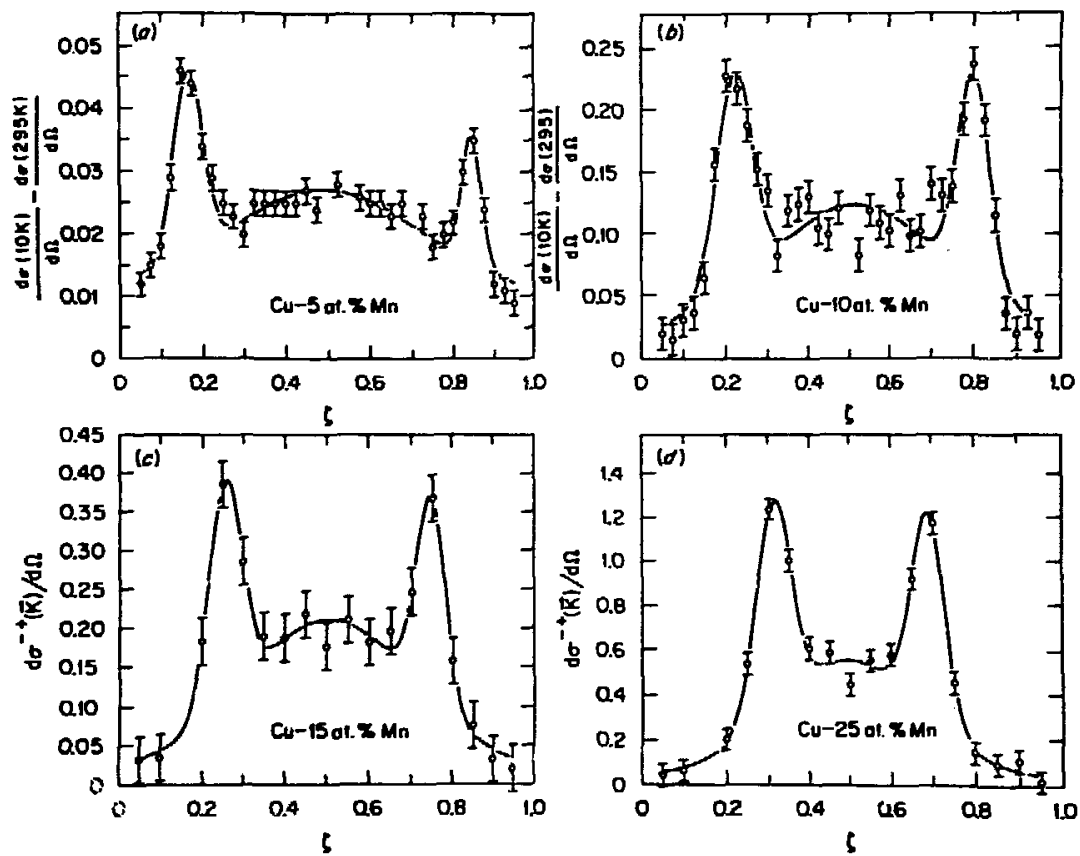

Flgure 2. Magnetic diffuse scattering cross sections for Cur-Mn alloye along the $\left[\begin{array}{lll}1 & \zeta & 0\end{array}\right]$ direction. Temperature difference data are shown in (a) and (b), while polarization analysis data for $10 \mathrm{~K}$ are given $1 \mathrm{n}(\mathrm{c})$ and (d). 
Q. If these conditions are not satisfied, then the static spin-pair correlations can only be determined by measurement of the full $S(\vec{Q}, \omega)$ followed by an $\omega$ Integration at constant $\vec{Q}$.

The magnetic diffuse scattering observed [19] for Cu-Mn alloys is shown in figure 2. These are data obtained with a triple-axis spectrometer set for elastic scattering and with the nuclear-magnecic separation achieved by the temperature difference method for (a) and (b) and by polarization analysis for (c) and (d). These data show two quite distinct types of magnetic short range order (MSRO). The broad diffuse peaks at $(11 / 20)$ artse fron a net ferromagnetic spln correlation that is directly associated wth the ASRO. The relat1vely sharp, but st111 diffuse, peaks at $(1,1 / 2 \pm \delta, 0)$ result from an antiferromagnetic spin correlation which takes the form of an incommensurate long period modulation. The modulation wavelength varles linearly with Mn concention in such a manner that $\delta$ approaches zero at $50 \% \mathrm{Mn}$. Th1s long perlod modulation has been attributed to an RKKY interaction between the Mn spins through the conduction electrons $[17,19]$. In th1s model, $\delta 1 \mathrm{~s}$ determined by a wavevector connecting approximately flat reglons of the Fermi surface.
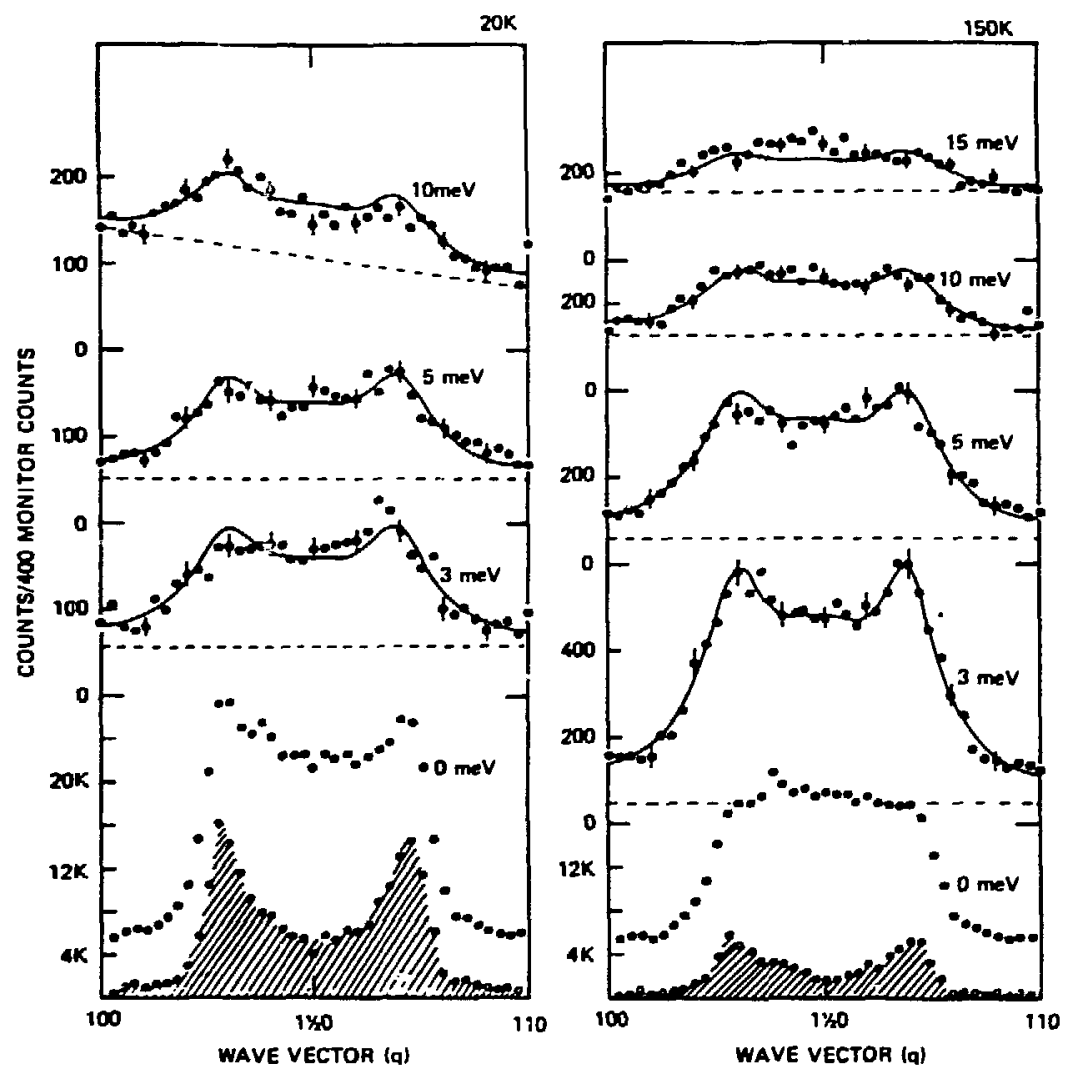

FIgure 3. Constant energy scans along $\left[\begin{array}{lll}1 & \zeta & 0\end{array}\right]$ for a Cu-217 Mn alloy at $20 \mathrm{~K}$ and $150 \mathrm{~K}\left(\mathrm{~T}_{\mathrm{f}}=90 \mathrm{~K}\right)$. The shaded areas represent magnetic scattering as obtalned by the temperature difference method. 
The microscoplc spin structure that emerges from these studies is that of small ferromagnetically correlated reglons homogeneously coexisting with the larger antiferromagnetic modulated regions. The spin dynamics associated with the ferromagnetic correlations was thoroughly studied by Murani [23-30] who found a wide spectral distribution of relaxation times evolving continuously with temperature. We [31] studied the spin dynamics of the antiferromagnetic correlations to better understand the interactions within and between these two types of MSRO regions. Figure 3 shows constant energy scans in the $\left(\begin{array}{ll}1 & 1 / 2\end{array}\right)$ region for a Cu-21\% Mn alloy at temperatures above and below the spin glass freezing temperature $\left(T_{f}=90 \mathrm{~K}\right)$. These are unpolarized-beam tripleaxis measurements with the final energy fixed at $13.8 \mathrm{mev}$ and with an energy resolution of 1.2 mev FWHM at zero energy transfer. The intense peak at $T=$ $20 \mathrm{~K}$ and $\Delta \mathrm{E}=0 \mathrm{mev}$ is a superposition of the MSRO peaks and the broad ASRO peak centered at $(1 \quad 1 / 20)$. The open data points and the shaded area represent an approximation to the magnetic diffuse scattering as obtained by the temperature difference method $(20 \mathrm{~K}-290 \mathrm{~K})$. This agrees well with the earlier measurements of figure 2. Even though $\mathrm{T} \ll \mathrm{T}_{f}$, inelastic scattering is observed with energy transfers up to $10 \mathrm{mev}$ and with the same peak positions as for the elastic scattering. Comparison of the $20 \mathrm{~K}$ and $150 \mathrm{~K}$ panels shows an intensity shift from elastic to inelastic scattering with increasing $T$. This continues until all of the scattering is inelastic at $290 \mathrm{~K}$ under the energy resolution condition of this experiment. The curves in figure 3 were fitted by assuming Lorentzian line shapes in both $\vec{Q}$ and $\omega$. The Inverse correlation length is observed to be temperature independent suggesting spin clusters that maintain approximately the same dimensions over the temperature Interval from $20 \mathrm{~K}$ to $290 \mathrm{~K}$. This study shows that the composite ferroantiferromagnetic clusters maintain their spatial correlations while undergoing an orlentational fluctuation in time. This is interesting physics, but for our purpose here, it serves to demenstrate that the inherent inelasticity of paramagnetic scattering can become a problem in attempted studies of static spin correlations. The validity of the quasi-elastic approximation must be carefully considered for each experiment.

\section{D1ffuse Scattering From Ni-Mn Alloys}

The magnetic behavior of N1-Mn alloys is also dominated by the presence of competing Interactions, but the holes in the $\mathrm{Ni} d$-band now allow for magnetic moments on the $\mathrm{N} 1$ sites. The $\mathrm{N} 1-\mathrm{r} 1 \mathrm{ch}$ alloys are ferromagnetic and exhibit a remarkable dependence of magnetization on both concentration and heat treatment. Ferromagnet1sm is retained to about $40 \% \mathrm{Mn}$ for annealed alloys but only to $24 \% \mathrm{Mn}$ for quenched alloys $[32,33]$. The overall behavior can be explained by a molecular fleld model which assumes nearest-neighbor exchange interac tions that are antiferromagnet1c for Mn-Mn pairs and ferromagnetic for $N 1-N 1$ and N1-Mn pairs. The alignment of the magnetic moment at a given Mn site then depends on the net effective fleld exerted by the nearest-neighbor environment of that site. Spin-reversal [34] and canted-sp1n [35] models have been suggested and either w111 reproduce the concentration dependence of the magnetization with properly selected parameters.

These models galn support from polarized-neutron diffuse scattering measurements [36] which show a pronounced local environment effect that is negative for first nelghbors and positive for second nelghbors. Also, NMR measurements 

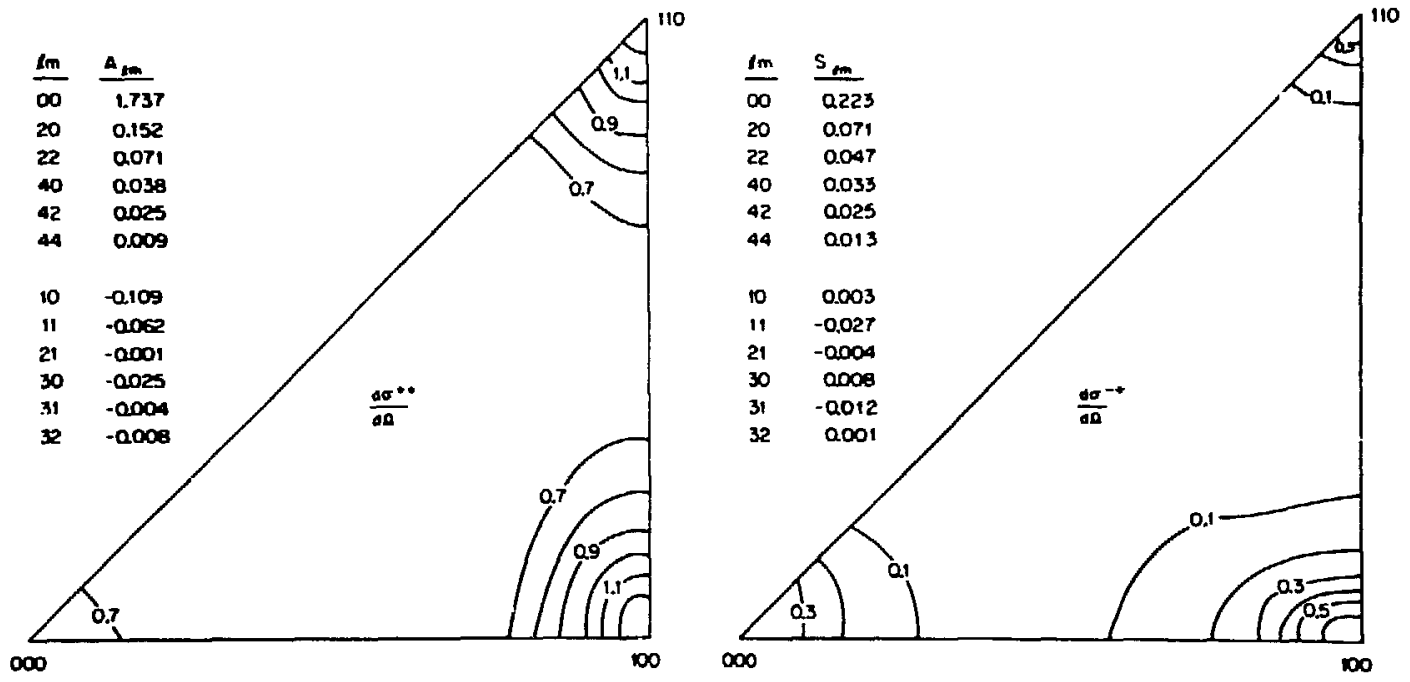

Figure 4. Contours of equal cross section for non-spin-filp (left) and spinflip (right) scattering from a N1-24\% Mn alloy. Contours are in units of barns-steradian-1 - atom ${ }^{-1}$. The $A_{\ell m}$ and $S_{\ell m}$ are two dimensional Fourler coefficients obtained from these data (see text).

[37] show a hyperfine field distribution at ${ }^{55} \mathrm{Mn}$ sites corresponding to three different types of Mn atoms. For quenched alloys, these competing interactions tend to cancel in the $24 \% \mathrm{Mn}$ region where ferromagnetism no longer exists. Just below $24 \%$ Mn reentrant spin-glass behavior 1s observed [38-40]. The reentrant state appears to be a mixed state with both a spontaneous ferromagnetic moment and spin glass characteristics. This mixed state interpretation is supported by inelastic neutron measurements [41] on a $22 \%$ Mn alloy which show long wavelength spin waves present both above and below the reentrant state phase boundary. He recently completed a polarization analysis study [42] of the atompair and spin-pair correlations in this reentrant state region. Diffuse scattering results for a $N 1-24 X$ Mn alloy are given in figure 4 where contours of equal cross section are shown on the left for non-spin flip scattering and on the right for spin-flip scattering. The nuclear scattering exhibits diffuse peaks at (100) and (110) which are associated with ASRO of the $\mathrm{Cu}_{3} \mathrm{Au}$ type. These data were Fourler transformed to obtain the two dimensional Fourler coefficlents listed alongside the figure. These are related to the three dimensional $\alpha(\vec{R})$ by

$$
A_{\ell m}=\sum_{n} a_{\ell m n} .
$$

Here, the atomic positions are given by

$$
\vec{R}=1 / 2\left(2+\vec{a}_{1}+\overrightarrow{m a}_{2}+\overrightarrow{n \vec{a}_{3}}\right)
$$

where $\ell$, $m$, and $n$ are integers and $(\ell+m+n)$ is even in this fcc lattice. 
The $A_{\ell m}$ have significant magnitudes out to $A_{4}$ is which contains contriburions from the $\alpha_{\ell m n}$ out to at least 16 or 18 shells. With ASRO to such large dis tances, there is insufficient information to obtain a unique solution for the even shell a $\alpha_{\text {mnn }}$. However, a reasonable solution can be obtained by setting all $\alpha_{\ell m n}$ beyond the $18 \mathrm{th}$ shell to zero and by constraining the $\alpha_{\operatorname{lmn}}$ to decrease with increasing $R$. The $\alpha_{\ell m n}$ obtalned with these restrictions show a preference for unlike odd-shell nelghbors and like even-shell nelghbors in accordance with the $\mathrm{Cu}_{3} \mathrm{Au}$ type of ASRO.

The spin-flip cross section in figure 4 shows similar features with diffuse peaks at $(000),(100)$, and $(110)$. In this experiment, the applied magnetic fleld (which is parallel to $\bar{Q}$ ) defines the $z$ axis of the spln system. Under these conditions, only the transverse spin components are observed so that the Fourier coefficients listed in the figure are given by

$$
S_{\ell m}=\sum_{\mathrm{n}} S_{\ell m n}
$$

where the MSRO parameter is

$$
s_{\ell m n}=\left\langle s_{n+R}^{x}+\underset{n}{\stackrel{x}{+}}\right\rangle \text {. }
$$

Three dimensional $S_{2 m n}$ were obtalned by using the same constralnts as those placed on the $\alpha_{\ell m n}$. These are positive for the even-shells and small but slightly negative for the first two odd-shells. This same alternating sequence was prevlously observed [36] for the longltudinal spln correlations, which suggests that spin-canting may be the correct model for these alloys.

IV. Diffuse Scattering From Au-Fe Alloys

The magnetic phase diagram of AuFe alloys exhibits a critical concentration, $c_{0}$, for the onset of ferromagnet $18 m$ near 16 at $\% \mathrm{Fe}$. At concentrations below $c_{D}$, there is a single boundary between paramagnetic and spin-glass phases, while above $c_{0}$, there is a double transition with a reentrant spin-glass phase $[43,44]$. It is generally accepted that this behavior is assoclated with ferromagnetic spin clusters, but there is considerable current controversy regarding the nature and interaction of these clusters near $c_{0}[45-47]$. Early interpretations of the double transition were based on a percolation model wh ferromagnetism assoclated wth the infinite cluster and spin-glass behavior attributed to coexistent finite clusters [43,44]. More recently, Mössbauer data [48-50], which show a canting of the moments away from the applied field direction in the reentrant phase, have been interpreted in terms of the mean fleld model of Gabay and Toulouse [51]. Th1s model 1s characterized by the coexistence of spontaneous ferromagnetism with spin-glass ordering of the transverse spin components.

$X$-ray diffuse scattering from quenched AuFe alloys in this critical composition region shows diffuse streaks in $\langle 210\rangle$ directions [52]. These are attributed to a tendency toward the formation of Fe atom platelets in $\{420\}$ planes which are approximately two atomic layers thick and $30 \mathrm{~A}$ in diameter. The authors speculate that the spins in these platelets are ferromagneticaliy aligned with the moments constrained to lie within the $\{420\}$ planes by shape antsotropy. Beck [53] reviews the experimental evidence and concludes that 


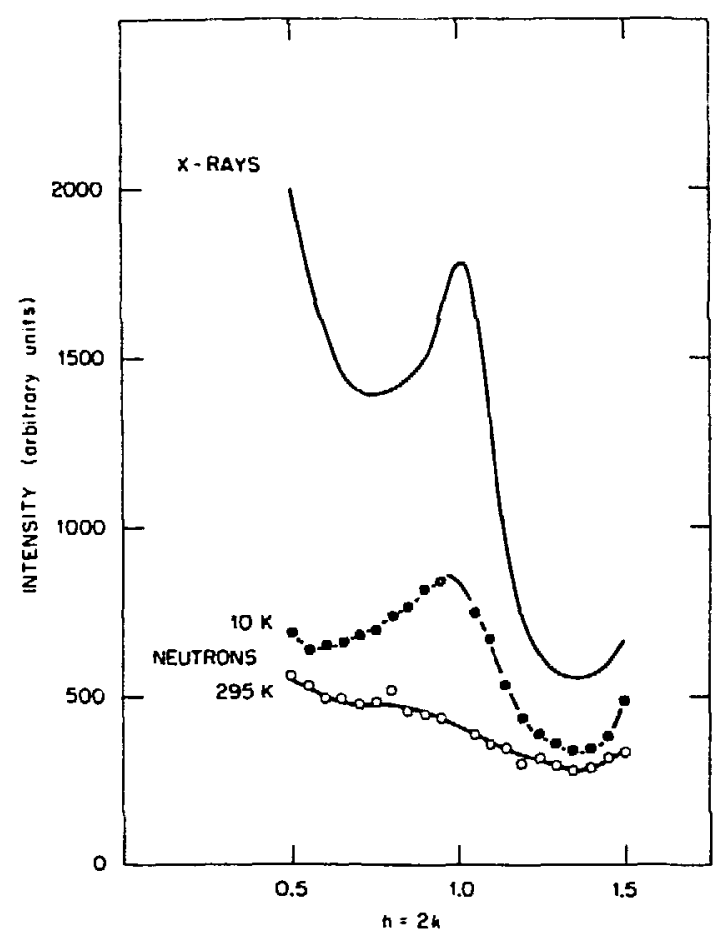

Figure 5. A comparison of the diffuse scattering in the [210] direction of $x$-rays [52] and of neutrons [54] from a Aur-15\% Fe alloy.

the magnetic behavior of these alloys is consistent with the presence of such platelets with the orientation of the platelet moment at low temperatures being determined by a quasi-random local anisotropy.

In view of the uncertainties in the nature of the spin correlations in these alloys, we decided to investigate these by neutron diffuse scattering [54]. Unpolarized neutrons were used and no separation of the nuclear and magnetic scatterlag was made. However, the expected Laue monotonic cross section for magnetic scattering $18109 \mathrm{f}(Q) \mathrm{mb} / \mathrm{atom}$ compared to only $5 \mathrm{mb} / \mathrm{atom}$ for the nuclear scattering. In essence then, the neutron cross section measures only the MSRO while the $x$-ray scattering determines the ASRO. A comparison of the $x$-ray and neutron results is given in figure 5 which shows the intensity measured along the [210] direction by the two techniques. The upper curve 1s a sketch of the x-ray data taken from reference [52], and the lover curves represent the neutron data at temperatures of $10 \mathrm{~K}$ and $295 \mathrm{~K}$. The magnet1c neutron scattering 18 clearly temperature dependent and peaks at $\left(\begin{array}{lll}1 & 1 / 2 & 0\end{array}\right)$ just as does the nuclear x-ray scattering. The simllarity between the $x$-ray and neutron data 18 even wore pronounced in two dimensional representation as given In figure 6 which shows 1 solntensity contours for the $T=10 \mathrm{~K}$ neu tron data. Just as for the $x$-ray case, there is diffuse scattering around the origin and a diffuse streak in the [210] direction. In addition, the Lorentzian half-wdth of the diffuse streak transverse to [210] Is the same 


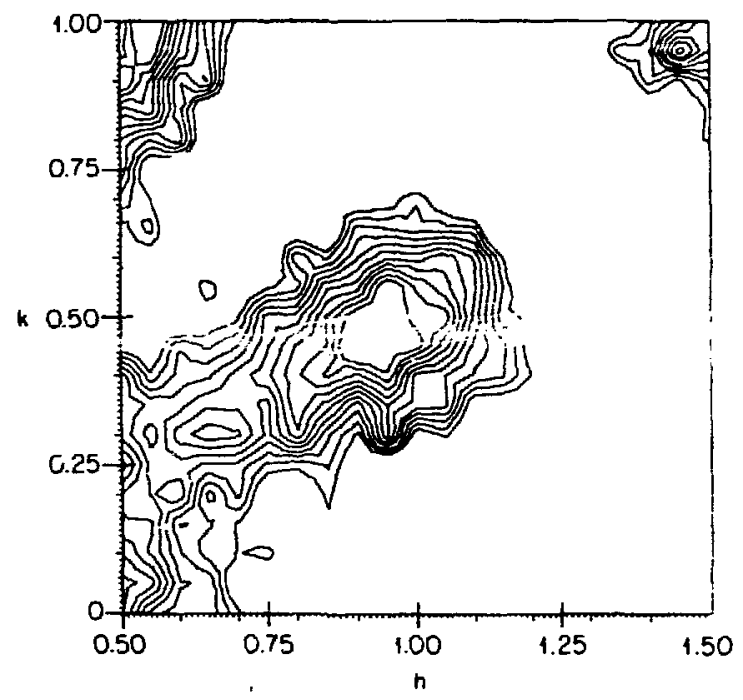

Figure 6. Isointensity contours for a Au-15\% Fe alloy at $10 \mathrm{~K}$. The contours are equally spaced from 550 to 800 counts.

$(0.1252 \pi / a)$ as that measured with $x$-rays. This similarity between the spinpair and atompair correlations is a consequence of the fact that only the Fe atoms have spins. The spin-pair correlation is then

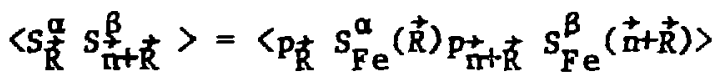

and the $\mathrm{Fe}-\mathrm{Fe}$ atom-pair correlation is

$$
\left\langle\mathrm{p}_{\mathrm{R}} \mathrm{p}_{\mathrm{n}+\mathrm{R}^{+}}^{+}\right\rangle=c^{2}+c(1-c) \alpha(\overrightarrow{\mathrm{R}}) \text {. }
$$

If these correlations are separable, then

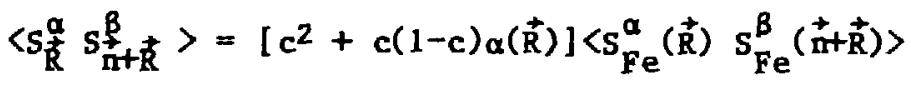

and a ferromagnetic Fe-Fe correlation will produce the observed low tem perature features of diffuse scattering at both $(000)$ and $(1 / / 20)$.

Additicaal Information about the nature of these correlations is gained from the effects of lattice distortions on the scattered intensity. This is quite pronounced in the $x$-ray case for which there is an asymetry in the streak Intensity around the fundamental reciprocal lattice points such that intensity is only observed for those streaks directed back toward the origin. This is expected if those atoms within the platelets have both a smaller scattering 
amplitude and a smaller atomic size than the average atom. In the Fe atom platelet model proposed by Dartyge et. al. [52], it is the intersection and superposition of intensity for four such streaks loriginating from (000), $(200),(111)$, and $11 \overline{1})]$ that produces the $(11 / 20)$ diffuse peak. However, the reverse situation should apply to the neutron data; 1.e., the smaller Fe atoms have the larger magnetic scattering amplitudes and diffuse streaks due to ferromagnetic platelets should point away from the origin. In that event, there should be no intersection of streaks and no diffuse peak at $(1 / 20)$. Tharefore, scattering from independent ferromagnet1c platelets cannot prouuce the observel neutron data which instead requires some type of damped periodicity in the direction normal to the platelets. We conclude that there is a tendency toward the formation of Fe platelets in $\{420\}$ planes; these are not randomly uistributed but have a quasi-periodicity in directlovs normal to the platelets. The MSRO is attributed to ferronagnetic spin corralations within these platelets but with a range that extends beyond that of the ASRO at low temperatures.

\section{Conclustons}

Neutron diffuse scattering measuraments have furnished a wealth of information on the magnetic moment fluctuations in magnetic materials. Most of the experfmental studies have dealt with the ferromagnetic substitucional alloys, and for these, the observed magnetic mowent spatial distributions have strongly impacted theoretical developments. The average magnetic moments of the constituents are now successfully calculated by the single-site CPA method and some of the observed local environment efrects are being obtained by cluster CPA calculations [55,56]. Such direct contact with theory is not yet possible for studies of paramagnetic systers. Nevertheless, much of the experimental activity has recently shifted into this area where spin correlation information is reeded to understand the behavior of new materials. Currently, these new materials include the reentrant spin glasses, the diluted magnetic semiconductors, and the high temperature superconductors. There w11l undoubtedly be other classes of materials for future studies. Such studies will produce even more detafled information than is presently obtained because of improvements in detection and data-handing systems, better polarized beam gethods, and the increasing avallabllity of single crystal sampies.

\section{Acknowledgments}

This research was supported by the Division of Materials Sclences, U. S. Department of Energy under contract DE-AC05-840R21400 with Martin Marietta Energy Systems, Inc. 
1. Halperin, 0. and Johnson, M. H.: Phys. Rev., 1939, 55, 898

2. Low, G. G.: Advances in Phys., 1969, 18, 371

3. Hicks, T. J.: "Treatise on Matertals Sclence and Technology," Vol. 15, Neutron Scattering, Academfic Preäs, New York, 1979, p. 337

4. Ralniord, B. D.: J. de Phys., 1982, 43, C7-33

5. Shull, C. G., Strauser, W. A., and Wollan, E. 0.: Phys. Rev., 1951, 83, 333

6. Beck, P. A.: Prog. Materials Science, 1978, 23, 1

7. MeneghettI, D. and Sidhu, S. S.: Phys. Rev., 1957, 105, 130

8. We11s, P. and Smlth, J. H.: J. Phys. F: Metal Phys., 1971, 1, 763

9. Sato, H., Werner, S. A., and Yess1k, M.: AIP Conf.Proc., 1971, 5, 508

10. Werner, S. A., Sato, H., and Yessik, M.: AIP Conf. Proc., 1972, 10, 679

11. Ahmed, N. and Hicks, T. J.: Solid Scate Commun., 1974, 15,415

12. Ahmed, N. and Hicks, T. J.: J. Phys. F: Met. Phys., 1975, 5, 2168

13. Davis, J. R. and Hicks, T. J.: J. Phys. F: Met. Phys., 1979, 9, 753

14. Dav18, J. R., Burke, S. K., and Rainford, B. D.: J. Magn. Magn. Mater., $1980,15-18,151$

15. Werner, S. A. and Cable, J. W.: J. Appl. Phys., 1981, 52, 1757

16. Cable, J. W., Werner, S. A., Felcher, G. P., and Wakabayash1, N.: Phys. Rev. Lett., $1982,49,829$

17. Harders, T. M. and Wells, P.: J. Phys. F: Met. Phys ., 1983, 13, 1017

18. Harders, T. M., Hicks, T. J., and Smith, J. H.: J. Phys. F: Met. Phys., $1983,13,1263$

19. Cable, J. H., Werner, S. A., Felcher, G. P., and Wakabayash1, N.: Phys. Rev. B, $1984,29,1268$

20. Werner, S. A. Rhyne, J. J., and Gotaas, J. A.: Solld State Commun., 1985, $\underline{56}, 457$

21. Gotaas, J. A., Rhyne, J. J., and Werner, S. A.: J. Appl. Phys., 1985, 57, 3404

22. Moon, R. M., Riste, T., and Koehler, W. C.: Phys. Rev., 1969, 181, 920

23. Murani, A. P.: J. Magn. Magn. Mater., 1977, 5, 95

24. Muran1, A. P.: Solid State Commun., 1977, 22, 25

25. Muran1, A. P. and Heldemann, A.: Phys. Rev . Lett., 1978, 41, 1402

26. Muran1, A. P.: Phys. Rev. Lett., 1978, 41, 1406

27. Meze1, F. and Muran1, A. P.: J. Magn. Magn. Mater., 1979, 14, 211

28. Murani, A. P.: Solid State Commun., 1980, 33, 433

29. Murani, A. P.: J. Magn. Magn. Mater., 1981, 22, 271

30. Muran1, A. P.: J. Phys. F: Met. Phys. 1985, 15,417

31. Tsunoda, Y., Kunitomi, N., and Cable, J. W.: J. Appl. Phys., 1985, 57, 3753

32. Kaya, S. and Kussuan, A.: Z. Physik, 1931, 72, 293

33. Plercy, G. R. and Morgan, E. R.: Can. J. Phys., 1953, 31, 529

34. Carr, H. J.: Phys. Rev., 1952, 85, 590

35. Sidorov, S. K. and Doroshenko, A. V.: Phys. Met. Metallogr., $1964,18,12$

36. Cable, J. W. and Child, H. R.: Phys. Rev. B, 1974, 10, 4607

37. Kltaoka, Y., Ueno, K., and Asayama, K.: J. Phys. Soc. Japan, 1978, 44, 142

38. Aftken, R. G., Cheung, T. D., Kouvel, J. S., and Hurdequint, J.: J. Magn. Magn. Mat., 1982, 30, L1

39. Abdu1-Razzaq, W. and Kouvel, J. S.: J. Appl. Phys., 1984, 55, 1623

40. Kouvel, J. S. and Abdul-Razzaq, W.: J. Magn. Magn. Mat., 1985, 53, 139 
41. Hennion, B., Hennion, M., Hippert, F., and Muran1, A. P.: J. Phys. F: Met. Phys.. 1984, 14, 489

42. Cable, J. W., Nicklow, R. M., and Tsunoda, Y.: Phys. Rev. B, sccepted for publication

43. Coles, B. R., Sarkisslan, B. V. B., and Taylor, R. H.: Phil. Mag. 1978, 37, 489

44. Verbeek, B. H. and Mydosh, J. A.: J. Phys. F: Met. Phys., 1978, 8, L109

45. Monod, P. and Campbel1, I. A. : Phys. Rev. Lett., 1984, 52, 2096

46. Brand, R. A. and Keune, W.: Phys. Rev. Lett., 1984, 52, 2097

47. V1olet, C. E. and Borg, R. J.: Phys. Rev. Lett., 1984, 52, 2098

48. Lauer, J. and Keune, W.: Phys. Rev. Lett., 1982, 48,1850

49. Varret, F., Hamzic, A., and Campbe11, I. A.: Phys. Rev. B, 1982, 26, 5285

50. Carpbe11, I. An, Senoussi, S., Varret, F., Tel11et, J., and HamzIc, A.: Phys. Rev. Lett., 1983, 50, 1615

51. Gabay, M. and Toulouse, G.: Phys. Rev. Lett., 1981, 47, 201

52. Dartyge, E., Bouchiat, H., and Monod, P.: Phys. Rev. B, 1982, 25, 6995

53. Beck, P. A.: Phys. Rev. B, 1985, 32, 7255

54. Cable, J. W., Parette, G., and Tsunoda, Y.: Phys. Rev. B, submitted for publication

55. Hamada, N.: J. Phys. Soc. Japan, 1981, 50, 77

56. Kakehash1, Y.: Phys. Rev. B., 1985, 32, 3035 